\title{
Temperature dependence and physical properties of Ga(NAsP)/GaP semiconductor lasers
}

\author{
J. Chamings, ${ }^{1}$ A. R. Adams, ${ }^{1}$ S. J. Sweeney, ${ }^{1, a)}$ B. Kunert, ${ }^{2, b)}$ K. Volz, ${ }^{2}$ and W. Stolz ${ }^{2}$ \\ ${ }^{1}$ Advanced Technology Institute, Faculty of Engineering and Physical Sciences, University of Surrey, \\ Guildford, Surrey GU2 7XH, United Kingdom \\ ${ }^{2}$ Material Sciences Center and Department of Physics, Philipps-University, Hans-Meerwein-Strasse, \\ D-35032 Marburg, Germany
}

(Received 8 May 2008; accepted 31 July 2008; published online 11 September 2008)

\begin{abstract}
We report on the properties of GaNAsP/GaP lasers which offer a potential route to producing lasers monolithically on silicon. Lasing has been observed over a wide temperature range with pulsed threshold current density of $2.5 \mathrm{kA} / \mathrm{cm}^{2}$ at $80 \mathrm{~K}(\lambda=890 \mathrm{~nm})$. Temperature dependence measurements show that the radiative component of the threshold is relatively temperature stable while the overall threshold current is temperature sensitive. A sublinear variation of spontaneous emission versus current coupled with a decrease in external quantum efficiency with increasing temperature and an increase in threshold current with hydrostatic pressure implies that a carrier leakage path is the dominant carrier recombination mechanism. (C) 2008 American Institute of Physics. [DOI: 10.1063/1.2975845]
\end{abstract}

The dominance of silicon $(\mathrm{Si})$ for electronic and microelectronic circuit applications has lead to the search for monolithic optoelectronic integrated circuits (OEICs) on $\mathrm{Si}$ substrates. One of the key components of OEICs is a laser material for efficient light emission. However, the indirect band gap of Si has meant efficient light emission and gain have been difficult to achieve. Several strategies for producing lasers on silicon have been proposed, such as the "hybrid" laser," whereby an InP-based active region is wafer fused onto a silicon/silica based waveguide or utilizing the Raman effect with external optical pumping. ${ }^{2}$ However monolithic growth on a silicon substrate coupled with electrical injection has remained challenging. Growth of conventional direct III-V compound semiconductors directly onto $\mathrm{Si}$ is very difficult due to the formation of threading dislocations as a result of the large lattice mismatch. However, it has been shown that GaP can be grown without dislocations on $\mathrm{Si}$ due to the relatively small difference in lattice constant $\left[<0.4 \%\right.$ at room temperature (RT) ${ }^{3}{ }^{3} \mathrm{GaP}$ is itself an indirect band gap semiconductor, but a GaNAsP alloy with high As fractions and dilute $\mathrm{N}$ fractions (of $\sim 4 \%$ ) can form a direct band gap material approximately lattice matched to $\mathrm{GaP}$ and $\mathrm{Si}^{4}{ }^{4}$ Hence the realization of a GaP-based direct band gap semiconductor laser material on a silicon substrate can provide a realistic route toward monolithic laser sources for silicon-based OEICs. This is also another example of the potential for dilute nitride based materials in optoelectronic components.

In this letter, we investigate the properties of GaNAsP lasers grown on GaP substrates. Using high pressure and low temperature techniques we have probed the extent to which a two level band anticrossing (BAC) (Ref. 5) model may be used to describe this material and have investigated the degree to which different carrier recombination processes govern laser behavior.

\footnotetext{
a) Electronic mail: s.sweeney @ surrey.ac.uk.

${ }^{b)}$ Present address: NAsP III/V GmbH, D-35041 Marburg, Germany.
}

The samples studied were grown by metal organic vapor phase epitaxy (MOVPE) on a GaP substrate. They consist of a single $6 \mathrm{~nm} \mathrm{GaN}{ }_{0.04} \mathrm{As}_{0.8} \mathrm{P}_{0.16} 2.5 \%$ compressively strained quantum well (SQW) within two undoped $150 \mathrm{~nm} \mathrm{GaP}$ barrier/separate confinement layers. Optical confinement is provided by $1.8 \mu \mathrm{m}$ thick $\mathrm{Al}_{0.23} \mathrm{Ga}_{0.77} \mathrm{P}$ cladding layers doped $p\left(\mathrm{Zn}: 7 \times 10^{18} \mathrm{~cm}^{-3}\right)$ and $n\left(\mathrm{Te}: 2 \times 10^{18} \mathrm{~cm}^{-3}\right)$ forming the diode junction. The device stripes (50 and $100 \mu \mathrm{m}$ wide) were defined using regular masks and standard contacts were formed by deposition. The devices were measured as-cleaved with cavity lengths of $980 \mu \mathrm{m}$ and had an emission wavelength of $890 \mathrm{~nm}$ at $80 \mathrm{~K}$.

Temperature dependence measurements were performed with a closed cycle cryostat setup over the temperature range of $70-150 \mathrm{~K}$. The lasers were driven under pulsed operation with $100 \mathrm{~ns}$ long pulses at a duty cycle of $10 \mathrm{kHz}$ in order to minimize Joule heating effects. Threshold currents could not be measured above $150 \mathrm{~K}$ due to the current source limit of 4 A. Other studies have shown that similar structures can achieve electrically pumped lasing up to $278 \mathrm{~K}$ in pulsed mode ${ }^{6}$ and optically pumped gain at RT. ${ }^{7}$ The threshold current was measured from the facet light versus current characteristic. Furthermore, we investigated the temperature and current dependencies of the pure spontaneous emission spectra from which we extracted the radiative component of the threshold current (since the integrated spontaneous emission is proportional to the radiative current). To measure the spontaneous emission, we milled a window in the $n$-doped side of the devices using a focused ion beam technique and aligned an optical fiber to collect the spontaneous emission. ${ }^{8}$ Hydrostatic pressure measurements were also performed on the devices at $100 \mathrm{~K}$ in a $\mathrm{CuBe}$ pressure cell (with optical and electrical access), allowing pressures of 0-10 kbars using gaseous helium as the pressure medium. The application of high hydrostatic pressure causes an increase in the direct band gap. Furthermore, in "dilute nitride" materials such as those discussed here, high pressure can be used to vary the interaction between the nitrogen level(s) and the conduction band (CB) edge of the host matrix and is therefore a useful 


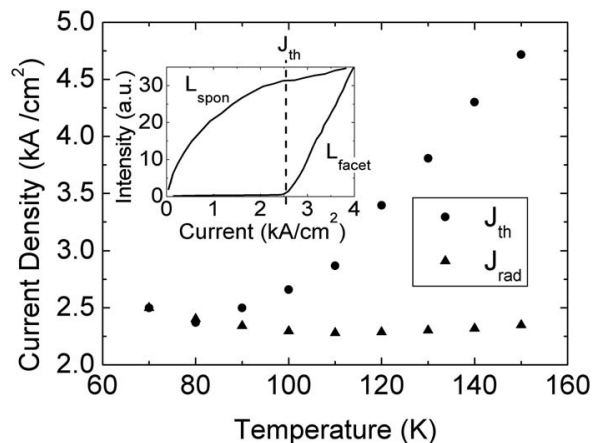

FIG. 1. $J_{\text {th }}$ (circles) and $J_{\text {rad }}$ (triangles) as a function of temperature (normalized at $70 \mathrm{~K}$ ). The inset shows the sublinear current dependence of $L_{\text {spon }}$ and the facet emission showing threshold (vertical dotted line) at $80 \mathrm{~K}$.

means of developing a further understanding of the behavior of such materials and the appropriateness of the BAC model.

In Fig. 1 we show the normalized (at $70 \mathrm{~K}$ ) temperature dependence of the threshold current density $J_{\text {th }}$ and its radiative component $J_{\text {rad. }}$. We find that these as-cleaved devices have threshold current densities of $2.5 \pm 0.5 \mathrm{kA} / \mathrm{cm}^{2}$ with a lasing wavelength of $890 \mathrm{~nm}$ at $80 \mathrm{~K}$. The inset shows the facet light versus current density characteristic and integrated spontaneous emission versus current density characteristic, both at $80 \mathrm{~K}$. This value of $J_{\text {th }}$ is much larger than well established GaAs-based lasers, which operate at similar wavelengths for which $J_{\text {th }} / \mathrm{QW} \sim 100-200 \mathrm{~A} / \mathrm{cm}^{2}$ (Ref. 9 and references therein), the cause of which became the focus of our investigations. The characteristic temperature $T_{0}$ [ $\left.=\left(d \ln J_{\mathrm{th}} / d T\right)^{-1}\right]$ was found to be $T_{0} \sim 200 \mathrm{~K}$ below $110 \mathrm{~K}$ dropping to $T_{0} \sim 60 \mathrm{~K}$ above $110 \mathrm{~K}$. Both $J_{\text {th }}$ and its radiative component $J_{\text {rad }}$ are unusually temperature stable below $110 \mathrm{~K}$, even higher than $T_{0}=T$, as would ideally be for a radiatively dominated quantum well. ${ }^{8}$ This may be an indication of inhomogeneities within the active region, as similar behavior (decreasing $J_{\text {th }}$ and $J_{\text {rad }}$ with increasing $T$ ) has been observed in other dilute nitride ${ }^{10}$ and quantum dot lasers ${ }^{11}$ where carrier localization due to material inhomogeneity can be significant. The lack of complete pinning of the spontaneous emission above threshold is also a signature of inhomogeneities. While $J_{\text {rad }}$ remains relatively stable over the temperature range studied, $J_{\text {th }}$ increases strongly above $110 \mathrm{~K}$. Therefore, it is clear that a nonradiative current increases with increasing temperature, causing $J_{\text {th }}$ to increase rapidly above $110 \mathrm{~K}$. The reduction in $T_{0}$ is an indication of a thermally activated recombination process such as carrier leakage. Auger recombination, which dominates lasers at longer wavelengths, ${ }^{8}$ is unlikely to be responsible at the short wavelengths and low temperatures considered here, as has previously been discussed in other materials at these wavelengths. ${ }^{12}$

The inset of Fig. 1 shows the current dependence of the integrated spontaneous emission below lasing threshold at 80 $\mathrm{K}$. The nonlinear behavior of the $L_{\text {spon }}$ versus current curve confirms that a nonradiative process is present. The fact that the curve is sublinear shows that this process has a stronger carrier density $(n)$ dependence than the radiative current (which itself has an approximately $\propto n^{2}$ dependence). ${ }^{8}$ Since carrier leakage has an approximately exponential dependence on $n$, this further suggests that this is the dominant recombination mechanism. We note here that recombination via defects $\left(\propto_{n}\right)$ in the QW is unlikely to be significant at threshold

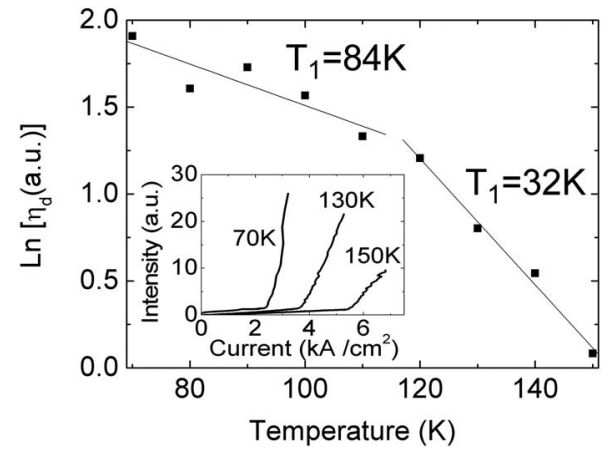

FIG. 2. The measured temperature dependence of slope efficiency yields a $T_{1}$ value of $84 \mathrm{~K}$ in the low $T$ range and $32 \mathrm{~K}$ at higher $T$. The inset shows typical $L-I$ curves at several temperatures.

as this would give rise to a superlinear variation of $L_{\mathrm{spon}}$ versus current curve (as observed in other materials ${ }^{10}$ ). Further evidence for carrier leakage can be obtained from the temperature variation of the external quantum efficiency, $\eta_{d}$, characterized by the $T_{1}$ parameter $\left[=\left(d \ln \eta_{d} / d T\right)^{-1}\right]$. A strong decrease in differential quantum efficiency, and hence low $T_{1}$ value, has previously been explained by an increase in leakage current to the $p$-cladding region. ${ }^{13}$ Indeed, when compared with other devices operating at similar wavelengths ${ }^{14}$ (for which $T_{1} \sim 1000 \mathrm{~K}$ around RT), we find $T_{1}$ to be somewhat low, $\sim 84 \mathrm{~K}$ for $T<100 \mathrm{~K}$ reducing to $\sim 32 \mathrm{~K}$ for $T>100 \mathrm{~K}$, as shown in Fig. 2.

Shown in Fig. 3 is the pressure dependence of the lasing energy (squares), taken from electroluminescence (EL) spectra of a GaNAsP laser, which increases with pressure at a rate of $5.0 \mathrm{meV} / \mathrm{kbar}$. Also shown is the pressure dependence of the $\Gamma$ minimum of the host material $\mathrm{GaAs}_{0.84} \mathrm{P}_{0.16}$, determined from a linear interpolation of GaAs and $\mathrm{GaP}$ (Ref. 15) which we find to be $10.2 \mathrm{meV} / \mathrm{kbar}$. Clearly the lasing energy has a much weaker pressure dependence than the direct band gap of the host GaAsP. It is therefore instructive to see if the BAC model can explain this reduced pressure dependence, as has been the case for other dilute nitride materials. ${ }^{16}$ In the BAC model, the interaction between a localized $N$ state and extended CB states splits the CB into two subbands, $E_{+}$and $E_{-}$. Their energies are described by the following equation:

$E_{ \pm}(P)=\frac{1}{2}\left\{\left[E_{\Gamma}(P)+E_{N}\right] \pm \sqrt{\left[E_{\Gamma}(P)-E_{N}\right]^{2}+4 x C_{M N}^{2}}\right\}$.

Here $E_{\Gamma}(P)$ is the host material pressure dependent $\Gamma \mathrm{CB}$ edge, $E_{N}$ is the energy level of the localized $N$ state, $x$ is the

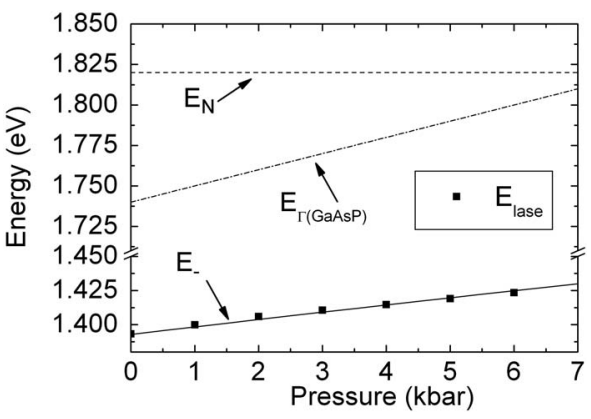

FIG. 3. Measured pressure dependence of the lasing energy (squares) for GaNAsP SQW lasers. Also shown is the pressure dependence of $\Gamma(\mathrm{GaAsP})$ (dotted-dashed line). The nitrogen level (dashed line) position was determined by interpolation of isolated $N$ level in GaAs and $\mathrm{GaP}$ and assumed negligible movement with pressure. The change in lasing energy with pressure can be predicted using the BAC model (solid line). 


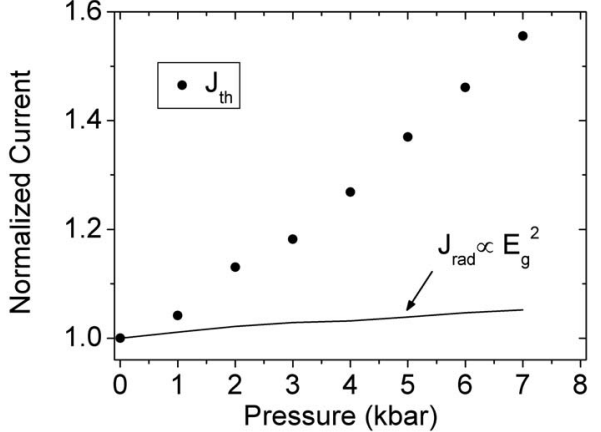

FIG. 4. Measured pressure dependence of $J_{\text {th }}$ and $J_{\text {rad }} \propto E_{g}^{2}$ for the GaNAsP devices, at $100 \mathrm{~K}$, normalized at atmospheric pressure.

$N$ fraction, and $C_{M N}$ is the coupling parameter determined by the strength of coupling between localized and extended states. All of these energies are defined with respect to the zone center valence band maximum. $E_{-}$is therefore taken as the "band gap" energy and compared to the lasing energy extracted from pressure dependent EL measurements. $E_{N}$ was found from interpolation of the isolated nitrogen level in $\mathrm{GaAs}$ and $\mathrm{GaP}$ and has been assumed to have a negligible pressure dependence. ${ }^{16}$ It is clearly seen in Fig. 1 that the BAC model gives good agreement with the experimental data for which $C_{M N}=1.9 \mathrm{eV}$. We note that this is similar to previous reported values for the GaInNAs/GaAs system for which $C_{M N}$ has been reported to range between 1.26 (Ref. 17) and $2.7 \mathrm{eV}$ (Ref. 18) depending on the composition of the host alloy and other factors.

Further evidence for the importance of nonradiative recombination may be found from high pressure measurements. Figure 4 shows the measured pressure dependence of $J_{\text {th }}$ for the GaNAsP devices at $100 \mathrm{~K}$. Also shown is the ideal expected variation ${ }^{12}$ of $J_{\mathrm{rad}} \propto E_{g}^{2}$ according to simple theory, where $E_{g}$ is taken to be the lasing energy (from $E_{\text {lase }}$ $=h c / \lambda_{\text {lase }}$, where $\lambda_{\text {lase }}$ is the measured lasing wavelength). Clearly, it can be seen that $J_{\text {th }}$ increases more rapidly with pressure than the ideal $J_{\text {rad }}$, showing an increase in nonradiative recombination with increasing pressure. This may occur if carriers escape into a leakage level, $E_{\text {leak }}$, which, in relative terms, moves closer to the $\mathrm{CB}$ edge with increasing pressure. Assuming diffusive leakage, the corresponding leakage current ${ }^{12}$ may be written as

$$
J_{\text {leak }}=J_{0} \exp \left(-\frac{d \Delta E}{d P} \frac{P}{k T}\right),
$$

where $J_{0}$ is a constant, $\Delta E$ is the energy separation between the CB quasi-Fermi level and the leakage level, $k$ is the Boltzmann constant, $T$ is the absolute temperature, and $P$ is the pressure. Thus, by considering the change in $\Delta E$ with pressure, $d \Delta E / d P$, the responsible leakage level may be identified from its pressure coefficient. From Fig. 4, a good fit is obtained for $d \Delta E / d P=-0.7 \mathrm{meV} / \mathrm{kbar}$. Since, from Fig. 3, the measured increase in band gap with pressure is $+5.0 \mathrm{meV} / \mathrm{kbar}$ and assuming that the $\mathrm{CB}$ quasi-Fermi level has similar pressure dependence, this corresponds to a pressure coefficient of $+4.3 \mathrm{meV} / \mathrm{kbar}$ for the leakage level. Hence we conclude that the leakage level has a slightly smaller pressure dependence than the $\mathrm{CB}$ quasi-Fermi level. This suggests that leakage into the $X$ minima of the indirect barriers (as previously observed in shorter wavelength GaNP light emitting diodes ${ }^{16}$ ) is not significant here, as the leakage level would then have a negative pressure coefficient.

In summary, we have found that the BAC model can well describe the band gap of $\mathrm{GaNAsP} / \mathrm{GaP}$ as a function of pressure. Furthermore, we have found that carrier leakage plays a significant role in GaNAsP lasers. A higher than expected $T_{0}$ at low temperatures is consistent with inhomogeneities in the active region. The sublinear dependence of the spontaneous emission on current and the reduction in both $T_{0}$ and $T_{1}$ at higher temperatures suggest that leakage currents are significant. If these leakage paths can be reduced, the growth of a direct band gap material on GaP and eventually Si substrates may lead to fully monolithic silicon-based OEICs operating at RT.

This project has been funded by the EPSRC (U.K.) and the Deutsche Forschungsgemeinschaft (DFG) within the Topical Research Group 483 "Metastable Compound Semiconductor Systems and Heterostructures."

${ }^{1}$ M. N. Sysak, H. Park, A. W. Fang, J. E. Bowers, R. Jones, O. Cohen, O. Raday, and M. J. Paniccia, Opt. Express 15, 15041 (2007).

${ }^{2}$ H. Rong, R. Jones, A. Liul, O. Cohen, D. Hak, A. Fangl, and M. Paniccia, Nature (London) 433, 725 (2005).

${ }^{3}$ H. Yonezu, Semicond. Sci. Technol. 17, 762 (2002).

${ }^{4}$ B. Kunert, K. Volz, J. Koch, and W. Stolz, Appl. Phys. Lett. 88, 182108 (2006).

${ }^{5}$ C. Skierbiszewski, Semicond. Sci. Technol. 17, 803 (2002).

${ }^{6}$ B. Kunert, A. Klehr, S. Reinhard, K. Volz, and W. Stolz, Electron. Lett. 42, 601 (2006).

${ }^{7}$ S. Borck, S. Chatterjee, B. Kunert, K. Volz, W. Stolz, J. Heber, W. W. Rühle, N. C. Gerhardt, and M. R. Hofmann, Appl. Phys. Lett. 89, 031102 (2006).

${ }^{8}$ S. J. Sweeney, A. F. Philips, A. R. Adams, E. P. O’Reilly, and P. J. A. Thijs, IEEE Photon. Technol. Lett. 10, 1076 (1998).

${ }^{9}$ G. Adolfsson, S. M. Wang, M. Sadeghi, and A. Larsson, Electron. Lett. 43, 454 (2007).

${ }^{10}$ R. Fehse, S. Tomic, A. R. Adams, S. J. Sweeney, E. P. O'Reilly, A. D. Andreev, and H. Riechert, IEEE J. Sel. Top. Quantum Electron. 8, 801 (2002).

${ }^{11}$ I. P. Marko, N. F. Massé, S. J. Sweeney, A. D. Andreev, and A. R. Adams, Appl. Phys. Lett. 87, 211114 (2005).

${ }^{12}$ D. Lock, S. J. Sweeney, A. R. Adams, and D. J. Robbins, Phys. Status Solidi B 235, 542 (2003).

${ }^{13}$ R. F. Kazarinov and M. R. Pinto, IEEE J. Quantum Electron. 30, 49 (1994).

${ }^{14}$ N. Tansu, Y.-L. Chang, T. Takeuchi, D. P. Bour, S. W. Corzine, M. R. T. Tan, and L. J. Mawst, IEEE J. Quantum Electron. 38, 640 (2002).

${ }^{15}$ S. Wei and A. Zunger, Phys. Rev. B 60, 5404 (1999).

${ }^{16}$ J. Chamings, S. Ahmed, S. J. Sweeney, V. A. Odnoblyudov, and C. W. Tu, Appl. Phys. Lett. 92, 021101 (2008).

${ }^{17}$ W. Shan, W. Walukiewicz, J. W. Ager III, E. E. Haller, J. F. Geisz, D. J. Friedman, J. M. Olson, and S. R. Kurtz, Phys. Rev. Lett. 82, 1221 (1999).

${ }^{18}$ P. Perlin, P. Wisniewski, C. Skierbiszewski, T. Suski, E. Kaminska, S. G. Subramanya, E. R. Weber, D. E. Mars, and W. Walukiewicz, Appl. Phys. Lett. 76, 1279 (2000). 